\title{
COMPACT SETS DEFINABLE BY FREE 3-MANIFOLDS
}

BY

\author{
W. H. ROW, JR. $\left.{ }^{1}\right)$
}

\begin{abstract}
Shape conditions are given that force a compactum (i.e., a compact metric space) embedded in the interior of a nonclosed, piecewise-linear 3manifold to have arbitrarily close, compact, polyhedral neighborhoods each component of which is a 3-manifold with free fundamental group (i.e., to be definable by free 3-manifolds). For compact, connected ANR's these conditions reduce to the criterion of having a free fundamental group. Additional conditions are given that insure definability by handlebodies or cubes-with-handles. An embedding of Menger's universal 1-dimensional curve in Euclidean 3-space is shown to have the property that all tame surfaces, separating in 3-space a fixed pair of points, cannot be adjusted (by a small space homeomorphism) to intersect the embedded curve in a 0 -dimensional set.
\end{abstract}

1. Introduction. Let $X$ be a compact metric space and let $b$ be a topological embedding of $X$ into the interior of a nonclosed, piecewise-linear 3-manifold $M^{3}$. We say $h(X)$ is definable by free 3-manifolds if $h(X)=\bigcap_{i=1}^{\infty} H_{i}$ where $H_{i}$ is a compact polyhedron in Int $M^{3}$ (the interior of $M^{3}$ ), each component of $H_{i}$ is a 3manifold with free fundamental group, and $H_{i+1} C$ Int $H_{i}$. Keep this notation fixed throughout the introduction.

In general the embedding $b$ may determine if $b(X)$ is definable by free 3 -manifolds. (See the examples in [23], [4], [5].) This paper gives shape properties of the components of $X$ that imply $h(X)$ is definable by free 3-manifolds. ( $\$ 4$, which deals with properties of special embeddings of 1-dimensional compacta, is an exception.) Our properties (uv( $r, s ; G)$ and UVF defined in $\$ 2$ ) fall under the category of "UV" or shape properties. We have chosen to define them in shape terms (i.e., ANR-sequences) rather than by neighborhood pairs (as in [19]). For embeddings in 3-manifolds these alternatives are equivalent.

Received by the editors March 22, 1973 and, in revised form, November 23, 1973. AMS (MOS) subject classifications (1970). Primary 57A10; Secondary 54F50, 55A99.

Key words and phrases. 3-manifold, handlebody, cube-with-handles, definable by free 3 -manifolds, definable by thin handlebodies, arc pushing properties, UV properties, ANRsequence, fundamental shape, fundamental domination, free group.

(1) The results in this paper are generalizations of portions of the author's Ph. D. thesis, University of Wisconsin, 1969, written under the direction of D. R. McMillan, J r. The research was partially supported by NSF Grants GP-7085, GP-19853, and GP-33872. 
Let us name some special 3-manifolds with free fundamental group. A compact, piecewise-linear 3-manifold $H^{3}$ is a bandlebody if $H^{3}$ is a regular neighborhood of a finite, polyhedral, connected graph in Int $H^{3}$. An orientable handlebody is a cube-with-bandles. We say $b(X)$ is definable by cubes-with-bandles if $b(X)$ is definable by free 3 -manifolds and in addition each component of $H_{i}$ (in the definition of definable by free 3-manifolds) is a cube-with-handles. Similarly we can define definable by handlebodies, definable by 3-cells, etc.

McMillan has studied the property of being definable by cubes-with-handles rather extensively [16], [17], [18], [19], [20], [21], [22]. If $M^{3}$ is orientable and $X$ is a 1-dimensional ANR [1], [16], a polyhedron collapsible to a 1-dimensional subpolyhedron [16], a $U^{\infty}$ set [19], or a Sierpiński curve [22], then $b(X)$ is definable by cubes-with-handles. Theorem 8.1 of Craggs [6] implies that if $X$ is a polyhedron each component of which has free fundamental group, then $b(X)$ is definable by free 3-manifolds. (Craggs actually obtains a much stronger result.) McMillan [17] had previously shown that if $X$ is a 2-sphere then $b(X)$ is definable by free 3-manifolds. In [18], [19] McMillan provides acyclicity conditions that insure $b(X)$ is definable by homotopy cubes-with-handles.

Theorem 15, and Corollaries 14,16,17, 19, 20 contain the results alluded to in the abstract. Our main result, Theorem 15, can be regarded as a generalization of [18, Theorem 3]. If we just consider the question of $b(X)$ being definable by free 3-manifolds, McMillan's results on planar compacta [22] are the only results mentioned that lie outside the scope of Theorem 15.

Let $n$ be a positive integer. If the components of $X$ are 2-spheres, diadic solenoids, toroidal continua, or are definable by cubes with no more than $n$ handles, then Theorem 15 implies that $h(X)$ is definable by free 3-manifolds. Another special case of the following question is answered in [22]. If each component of a compact set $Y$ embedded in the interior of a 3-manifold is definable by free 3-manifolds, is $Y$ definable by free 3 -manifolds?

We will assume the reader is acquainted with regular neighborhood theory [28] and geometric group theory [12], [15]. A good reference for algebraic topology is [25].

We will use $Z_{p}, p$ a prime or zero, to denote the integers modulo $p\left(Z_{0}\right.$ is the integers). We only use $Z_{p}$ as coefficient group for homology groups. In a given context $p$ will remain fixed. (We also use $Z_{i}, Z_{0}, Z$ as names for polyhedra. There should be no confusion.)

We adopt the convention that unidentified arrows between homology or homotopy groups will denote inclusion induced homomorphisms. If $A \rightarrow B$ is a homomorphism we denote the kernel and image of $A \rightarrow B$ by $\operatorname{ker}[A \rightarrow B]$, image $[A \rightarrow B]$, respectively. We use $\rho G$ to denote the rank of a group $G$ (see $\$ 2$ for the definition). Manifolds are always connected, separable, metric spaces. We use $\partial M$ to denote the boundary of a manifold $M$. 
2. Shape properties. In this section we will show properties uv $(r, s ; G)$ and UVF, defined below, are shape properties for compact metric spaces. We will use the ANR-sequence approach to shape developed in [13], [14]. See p. 61 of [13] for a complete set of the definitions involved and a comparison with Borsuk's definition of shape [3].

Let $X$ be a compact metric space. Then $\underline{X}=\left\{X_{n}, p_{n, n+1}\right\}$ is an ANR-sequence associated with $X$ if $\underline{X}$ is an inverse sequence of compact ANR's for metric spaces and $X$ is homeomorphic to the inverse limit of $\underline{X}$. We will suppress the homeomorphism between $X$ and the inverse limit of $\underline{X}$. A map of sequences $\underline{f}: \underline{X} \rightarrow \underline{Y}$ where $\underline{Y}=\left\{Y_{n}, g_{n, n+1}\right\}$ consists of an increasing function $f: N \rightarrow N(N$ is the natural numbers) and a collection of maps $f_{n}: X_{f(n)} \rightarrow Y_{n}$ such that $f_{n} p_{f(n), f\left(n^{\prime}\right)}$ is homotopic to $g_{n, n^{\prime}} f_{n^{\prime}}$ for $n \leq n^{\prime}$. Two maps $\underline{f}, \underline{g}: \underline{X} \rightarrow \underline{Y}$ are bomotopic, $\underline{f} \simeq \underline{g}$, pro-

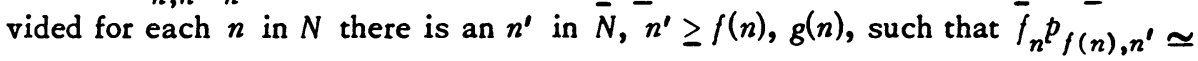
$g_{n} p_{g(n), n^{\prime}}$. The composite $\underline{g f}: \underline{X} \rightarrow \underline{Z}$ of $\underline{f}: \underline{X} \rightarrow \underline{Y}$ and $\underline{g}: \underline{Y} \rightarrow \underline{Z}$ is a map of sequences $\underline{b}: \underline{X} \rightarrow \underline{Z}$ given by $b=f g: \bar{N} \rightarrow N$ and $b_{n}=g_{n} f_{g(n)}: X_{f g(n)} \rightarrow Z_{n}$. The identity map of sequences $\underline{1}_{\underline{X}}: \underline{X} \rightarrow \underline{X}$ is given by $1_{N}: N \rightarrow N$ and $1_{X_{n}}: X_{n}$ $\rightarrow X_{n}$. Two metric compacta $X$ and $Y$ have the same sbape, $\operatorname{Sh}(X)=\operatorname{Sh}(Y)$, provided for some ANR-sequences $\underline{X}$ and $\underline{Y}$ associated with $X$ and $Y$, respectively, there exist maps of sequences $\underline{f}: \underline{X} \rightarrow \underline{Y}$ and $\underline{g}: \underline{Y} \rightarrow \underline{X}$ such that $\underline{g} \underline{\simeq} \underline{1}_{X}$ and $\underline{f g} \simeq \underline{1}_{Y}$. We say $\operatorname{Sh}(X)$ fundamentally dominates $\operatorname{Sh}(Y)$, written $\operatorname{Sh}(X) \geq \overline{\operatorname{Sh}}(Y)$, if in the above definition of $\operatorname{Sh}(X)=\operatorname{Sh}(Y)$ we do not require $g f \simeq \underline{1}_{X}$. Borsuk defines $\mathrm{Sh}(X) \geq \mathrm{Sh}(Y)$ on p. 25 of [3] and the proofs in [13] show the definitions are equivalent for compact metric spaces. In [14] Mardešić and Segal show the notions of shape and fundamental domination do not depend on our choices of $\underline{X}$ and $\underline{Y}$ associated with $X$ and $Y$.

We will need the following items to define properties uv $(r, s ; G)$ and UVF.

If $A$ is a finitely generated group let the rank of $A$, written $\rho A$, be the least number of generators in a presentation of $A$. We will use the following properties of rank. (J. E. Leech provided the proof of (4).) (1) If $A$ is a finitely generated group and $f: A \rightarrow B$ is an epimorphism then $\rho B \leq \rho A$. (2) If $A$ and $B$ are finitely generated abelian groups and $f: A \rightarrow B$ is an epimorphism then $\rho A \leq \rho B+\rho$ ker $f$. (3) If $A$ is a finite dimensional vector space over $Z_{p}, p$ a prime, then $\rho A=\operatorname{dim} A$. (4) If $A$ is a finitely generated abelian group and $B$ is a subgroup of $A$ then $\rho B$ $\leq \rho A$. (Note that each epimorphism from the free group on $k$ elements onto $A$ factors through the free abelian group $G_{k}$ on $k$ elements. If we consider the preimage of $B$ in $G_{k}$, we see that $\rho B \leq k_{\text {.) }}$

If $d: A \rightarrow B$ is a group homomorphism we say $d$ factors through a free group if there exist a free group $F$ and homomorphisms $d_{1}: A \rightarrow F, d_{2}: F \rightarrow B$ such that $d_{2} d_{1}=d$. If $X$ is a topological space, frequently we will use a prime, $X^{\prime}$, to denote a component of $X$. If $f: X \rightarrow Y$ is a map then $f^{\prime}: X^{\prime} \rightarrow Y^{\prime}$ will 
denote the map $\left.f\right|_{X^{\prime}}: X^{\prime} \rightarrow Y^{\prime}$ where $Y^{\prime}$ is the component of $Y$ that contains $f\left(X^{\prime}\right)$.

Let $\underline{X}=\left\{X_{n}, p_{n, n+1}\right\}$ be an ANR-sequence associated with a compact metric space $X$. Let $G$ be a finitely generated Abelian group. Suppose $r$ and $s$ are integers, $r \geq 1$, and $s \geq 0$. We say $X$ has property uv $(r, s ; G)$ if given $k$ there exists $l$ such that if $X_{l}^{\prime}$ is a component of $X_{l}$ and $\left(p_{k, l}^{\prime}\right)_{*}: H_{r}\left(X_{l}^{\prime} ; G\right) \rightarrow H_{r}\left(X_{k}^{\prime} ; G\right)$ is the homomorphism induced by $p_{k, l}^{\prime}$, then

$$
\rho\left(p_{k, l}^{\prime}\right)_{*}\left(H_{r}\left(X_{l}^{\prime} ; G\right)\right) \leq s .
$$

$X$ has property UVF if given $k$ there exists $l$ such that if $X_{l}^{\prime}$ is a component of $X_{l}$ then the homomorphism $\left(p_{k, l}^{\prime}\right)_{\#}: \pi_{1}\left(X_{l}^{\prime}, x\right) \rightarrow \pi_{1}\left(X_{k}^{\prime}, p_{k, l}^{\prime}(x)\right)$, induced by $p_{k, l}^{\prime}$, factors through a free group. Note that any map $f: X_{l}^{\prime} \rightarrow X_{k}^{\prime}$ that is homotopic (not necessarily keeping $x$ fixed) to $p_{k, l}^{\prime}$ also induces a homomorphism that factors through a free group. Theorem 1 shows properties uv( $r, s ; G)$ and UVF do not depend on our choice of $\underline{X}$.

Remark. Suppose $b$ is a topological embedding of a compact metric space $X$ into the interior of a nonclosed, piecewise-linear 3-manifold $M^{3}$ and $H_{1}, \cdots, H_{n}$, ... are compact polyhedra in Int $M^{3}$ such that $b(X)=\bigcap_{n=1}^{\infty} H_{n}$, each component of $H_{n}$ is a 3-manifold, and $H_{n+1}$ C Int $H_{n}$. Let $b_{n, n+1}: H_{n+1} \rightarrow H_{n}$ denote the inclusion map. Our primary interest is in the ANR-sequence $\underline{H}=\left\{H_{n}, b_{n, n+1}\right\}$ associated with $X$.

Note our property uv $\left(n, 0 ; Z_{p}\right)$ is equivalent to McMillan's property $n-\operatorname{uv}\left(Z_{p}\right)$ [19] for embeddings in 3-manifolds. By using a $p$-adic ( $p$ a prime) solenoid type construction with an increasing number of handles we can find a continuum that has property uv $\left(1,0 ; Z_{p}\right)$ but not property $u v\left(1, k ; Z_{q}\right)$ for each $k$ and prime $q \neq p$.

Theorem 1. Properties uv $(r, s ; G)$ and UVF are shape properties and are preserved by fundamental domination.

Proof. It suffices to show our properties are preserved by fundamental domination. Suppose $\operatorname{Sh}(X) \geq \operatorname{Sh}(Y)$ where $X$ and $Y$ are compact metric spaces. Using the notation introduced in defining $\operatorname{Sh}(X) \geq \operatorname{Sh}(Y)$, we have maps of sequences $f$ : $\underline{X} \rightarrow \underline{Y}$ and $\underline{g}: \underline{Y} \rightarrow \underline{X}$ such that $\underline{f g} \simeq \underline{1}_{\underline{Y}}$. It follows from the definitions that given $k$, for all $l \geq f(k)$ there exists an $n \geq g(l)$ such that

$$
q_{k, n} \simeq f_{k} p_{f(k), l{ }_{l}{ }_{8}(l), n}
$$

(i.e., $q_{k, n}$ homotopy factors through $p_{f(k), l}$ ).

Suppose $X$ has property uv(r,s;G). Then given $k$, for some $l$ and all components $X_{l}^{\prime}$ of $X_{l}, \rho\left(p_{f(k), l}^{\prime}\right)_{*}\left(H_{r}\left(X_{l}^{\prime} ; G\right)\right) \leq s$. Hence there exists an $n \geq g(l)$ such 
that for each component $Y_{n}^{\prime}$ of $Y_{n}, \rho\left(q_{k, n}^{\prime}\right)_{*}\left(H_{r}\left(Y_{n}^{\prime} ; G\right)\right) \leq s$. So $Y$ has property uv $(r, s ; G)$.

Suppose $X$ has property UVF. Then given $k$, for some $l$ and all components

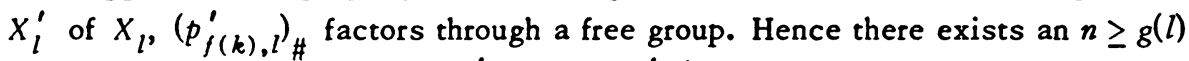
such that for each component $Y_{n}^{\prime}$ of $Y_{n},\left(q_{k, n}^{\prime}\right)_{\#}$ factors through a free group. Therefore $Y$ has property UVF.

An easy compactness argument yields

Lemma 2. Suppose $X$ is a compact metric space and $\mathrm{P}$ is one of the properties uv $(r, s ; G)$ or UVF. Then $X$ bas property $\mathrm{P}$ if and only if each component of $X$ has property $\mathrm{P}$.

Theorem 3. Suppose $X$ is a compact metric space and each component of $X$ has property uv $\left(2, s ; Z_{p}\right)$ where $p=0$ or a prime. Let $b$ be a topological embedding of $X$ into the interior of a nonclosed, piecewise-linear 3-manifold $M^{3}$. If $M^{3}$ is nonorientable assume $p=2$. Then $b(X)=\bigcap_{i=1}^{\infty} H_{i}$ where $H_{i}$ is a compact polyhedron in Int $M^{3}$, each component of $H_{i}$ is a 3-manifold with no more than $s+1$ boundary components, and $H_{i+1} \subset$ Int $H_{i}$.

Proof. Let $U$ be a neighborhood of $b(X)$ in Int $M^{3}$. It is sufficient to find a compact polyhedron $H \subset U$ such that each component of $H$ is a 3-manifold with no more than $s+1$ boundary components and $b(X) \subset$ Int $H$. Since $X$ has property uv $\left(2, s ; Z_{p}\right)$ we can find compact polyhedra $P$ and $R$ contained in $U$ such that each component of $P$ or $R$ is a 3-manifold, $P \subset$ Int $R, b(X) \subset$ Int $P$, and for each component $P^{\prime}$ of $P$ and $R^{\prime}$ of $R$ such that $P^{\prime} \subset R^{\prime}$ we have

$$
\rho \text { image }\left[H_{2}\left(P^{\prime} ; Z_{p}\right) \rightarrow H_{2}\left(R^{\prime} ; Z_{p}\right)\right] \leq s .
$$

Suppose $P^{\prime}$ is a component of $P$ and $P^{\prime}$ is contained in $R^{\prime}$, a component of $R$. Let $H^{\prime}$ be obtained from $P^{\prime}$ by adding to $P^{\prime}$ all components of the closure of $R^{\prime}-P^{\prime}$ that do not intersect $\partial R^{\prime}$, the boundary of $R^{\prime}$. Note $H^{\prime} \supset P^{\prime}, \partial H^{\prime} \subset \partial P^{\prime}$, each component of $R^{\prime}-H^{\prime}$ meets $\partial R^{\prime}$, and if $P^{\prime \prime}$ is another component of $P$ then $H^{\prime}$ contains $P^{\prime \prime}$ or $H^{\prime} \cap P^{\prime \prime}=\varnothing$.

$H^{\prime}$ has at most $s+1$ boundary components. To establish this claim let $S_{1}$, $\cdots, S_{r}$ be the boundary components of $H^{\prime}$. Let $J_{1}, \cdots, J_{r-1}$ be polyhedral arcs in $R^{\prime}$ such that $J_{i}$ pierces each of $S_{i}$ and $S_{r}$ in exactly one point, $J_{i} \cap S_{j}=\varnothing$ for $j \neq i$ or $r$, and $\partial J_{i} \subset \partial R^{\prime}$. There exist homomorphisms $d_{i}: H_{2}\left(R^{\prime} ; Z_{p}\right) \rightarrow Z_{p}$ given by computing the geometric intersection number $\bmod p$ of a representative of an element of $H_{2}\left(R^{\prime} ; Z_{p}\right)$ with $J_{i}$. Hence $S_{1}, \cdots, S_{r-1}$ are carriers of independent elements of $H_{2}\left(R^{\prime} ; Z_{p}\right)$. Since $\partial H^{\prime} \subset \partial P^{\prime}, r-1 \leq s$. Hence $r \leq s+1$ as claimed. If $H^{\prime}$ and $H^{\prime \prime}$ are obtained from components $P^{\prime}$ and $P^{\prime \prime}$ of $P$, respectively, as in the preceding two paragraphs then $H^{\prime} \cap H^{\prime \prime}=H^{\prime}, H^{\prime \prime}$, or $\varnothing$. Suppose not. Since 
$P^{\prime} \cap P^{\prime \prime}=\varnothing$ there exist components $Q^{\prime}$ of the closure of $R^{\prime}-P^{\prime}$ and $Q^{\prime \prime}$ of the closure $R^{\prime \prime}-P^{\prime \prime}$ such that $Q^{\prime} \cap Q^{\prime \prime} \neq \varnothing, \partial Q^{\prime} \subset P^{\prime}, \partial Q^{\prime \prime} \subset P^{\prime \prime}, P^{\prime} \subset Q^{\prime \prime}$, and $P^{\prime \prime} \subset Q^{\prime}$. Hence $Q^{\prime} \cup Q^{\prime \prime}$ is a closed 3-manifold contained in $M^{3}$, an impossibility.

Now a maximal disjoint collection of the 3 -manifolds $H^{\prime}$ will be the components of the required $H$.

3. Simple moves in 3-manifolds. In this section we will develop the algebraicgeometric background we need. This section relies heavily on results of D. R. McMillan, Jr.

We will assume the reader is familiar with McMillan's notation and results on simple moves in an orientable, nonclosed, piecewise-linear 3-manifold $M^{3}$ (see $\$ 2$ of [18] and [20]). If we remove the requirement that $M^{3}$ be orientable, only the definition of $c\left(Z^{3}\right)$, on p. 130 of [18] and p. 162 of [20], needs to be altered. Redefine $c\left(Z^{3}\right)=\Sigma(3-x(S))^{2}$ where the sum extends over all closed surfaces $S$ in $\partial Z^{3}$ and $x(S)$ is the Euler characteristic of $S$. Now McMillan's results in $\$ 2$ of [18] and $\$ 2$ of [20] hold for possibly nonorientable, nonclosed, piecewise-linear 3-manifolds $M^{3}$.

We will basically retain McMillan's notation in this section. Note 3-manifolds are connected and $M^{3}$ will always be a nonclosed, piecewise-linear 3-manifold. We deviate from McMillan's notation by using superscripts on 3-manifolds (i.e., $M^{3}$ ) and dropping them on compact polyhedra each component of which is a 3-manifold (i.e., $W_{i}$ instead of $W_{i}^{3}$ ). Recall that unidentified arrows denote inclusion induced homomorphisms.

Lemma 4. Let $p=0$ or a prime. Suppose $W_{1}, W_{2}, W_{3}$ are compact polybedra such that each component of $W_{i}$ is a 3-manifold, $W_{3} \subset$ Int $W_{2}$, and $W_{2} \subset$ Int $W_{1}$. Suppose that $W$ is obtained from $W_{2}$ by an annexation of type 2 or a simple reduction in $W_{1}$ and that $W_{3} \subset$ Int $W$. Let $W^{\prime}$ be a component of $W$ and suppose $W_{3}^{\prime} \subset$ $W_{2}^{\prime} \subset W_{1}^{\prime}$ are components of $W_{3}, W_{2}, W_{1}$, respectively, such that $W_{3}^{\prime} \subset W^{\prime}$. Then

$$
\rho \text { image }\left[H_{1}\left(W_{3}^{\prime} ; Z_{p}\right) \rightarrow H_{1}\left(W^{\prime} ; Z_{p}\right)\right] \leq \rho \text { image }\left[H_{1}\left(W_{3}^{\prime} ; Z_{p}\right) \rightarrow H_{1}\left(W_{2}^{\prime} ; Z_{p}\right)\right]
$$

and image $\left[H_{1}\left(W^{\prime} ; Z_{p}\right) \rightarrow H_{1}\left(W_{1}^{\prime} ; Z_{p}\right)\right]$ is a subgroup of image $\left[H_{1}\left(W_{2}^{\prime} ; Z_{p}\right) \rightarrow H_{1}\left(W_{1}^{\prime} ; Z_{p}\right)\right]$.

Proof. If $W^{\prime}=W_{2}^{\prime}$ we are done. So suppose $W^{\prime} \neq W_{2}^{\prime}$. If $W$ is obtained from $W_{2}$ by an annexation of type 2 then using the Mayer-Vietoris sequence of $W_{2}^{\prime}$ and the closure of $W^{\prime}-W_{2}^{\prime}$ with coefficients $Z_{p}[25, \mathrm{p} .218]$ we see that $H_{1}\left(W_{2}^{\prime} ; Z_{p}\right) \rightarrow$ $H_{1}\left(W^{\prime} ; Z_{p}\right)$ is an epimorphism. If $W$ is obtained from $W_{2}$ by simple reduction then using the Mayer-Vietoris sequence of $W^{\prime}$ and the closure of $W_{2}^{\prime}-W^{\prime}$ with coefficients $Z_{p}$ we see that $H_{1}\left(W^{\prime} ; Z_{p}\right) \rightarrow H_{1}\left(W_{2}^{\prime} ; Z_{p}\right)$ is a monomorphism. These two facts applied to the four appropriate diagrams yield the desired conclusions. 
Lemma 5 for orientable 3-manifolds is given in [21, Lemma 10.2]. We use essentially the same proof. It is included here for completeness.

Lemma 5. Let $\mathrm{M}^{3}$ be a compact 3-manifold with nonempty boundary. Let $p$ be a prime and if $M^{3}$ is nonorientable assume $p=2$. Then

$$
2 \rho \text { image }\left[H_{1}\left(\partial M^{3} ; Z_{p}\right) \rightarrow H_{1}\left(M^{3} ; Z_{p}\right)\right]=\rho H_{1}\left(\partial M^{3} ; Z_{p}\right) .
$$

Proof. Let $I=$ image $\left[H_{1}\left(\partial M^{3} ; Z_{p}\right) \rightarrow H_{1}\left(M^{3} ; Z_{p}\right)\right]$. Note $M^{3}$ is $Z_{p}$-orientable. The exact homology sequence $\left(Z_{p}\right.$ coefficients) of the pair $\left(M^{3}, \partial M^{3}\right)$ may be separated into two exact sequences thus:

$$
\begin{array}{cccc}
0 & \rightarrow H_{3}(M, \partial M) \rightarrow H_{2}(\partial M) & \rightarrow H_{2}(M) & \rightarrow H_{2}(M, \partial M) \rightarrow H_{1}(\partial M) \rightarrow I \rightarrow 0 \\
\downarrow & \downarrow & \downarrow & \downarrow \\
0 & \leftarrow H_{0}(M) \longleftarrow H_{0}(\partial M) \leftarrow H_{1}(M, \partial M) \longleftarrow H_{1}(M) \longleftarrow I \longleftarrow 0
\end{array}
$$

The vertical arrows represent isomorphisms given by Lefschetz duality [25, p. 298] and the universal coefficient theorem [25, p. 244]. By exactness the alternating sum of the ranks of the groups appearing in each row is zero. Hence our conclusion follows.

Corollary 6 for orientable 3-manifolds appears in [21].

Corollary 6. Suppose $T$ is a boundary component of a compact 3-manifold $M^{3}$. Let $p$ be a prime and if $M^{3}$ is nonorientable assume $p=2$. Then

$$
2 \rho \text { image }\left[H_{1}\left(T ; Z_{p}\right) \rightarrow H_{1}\left(M^{3} ; Z_{p}\right)\right] \geq \rho H_{1}\left(T ; Z_{p}\right) .
$$

Proof. In this proof homology groups will be with $Z_{p}$ coefficients. Let $D^{3}$ be the 3-manifold obtained by taking disjoint copies $M_{1}^{3}, M_{2}^{3}$, of $M^{3}$ and identifying corresponding boundary components by the identity map except for $T_{1}$ and $T_{2}$, the copies of $T$. Suppose $2 \rho$ image $\left[H_{1}(T) \rightarrow H_{1}\left(M^{3}\right)\right]<\rho H_{1}(T)$. Then

$$
\begin{aligned}
2 \rho \text { image }\left[H_{1}\left(T_{1} \cup T_{2}\right) \rightarrow H_{1}\left(D^{3}\right)\right] & \leq 4 \rho \text { image }\left[H_{1}\left(T_{1}\right) \rightarrow H_{1}\left(D^{3}\right)\right] \\
& \leq 4 \rho \text { image }\left[H_{1}(T) \rightarrow H_{1}\left(M^{3}\right)\right] \\
& <2 \rho H_{1}(T)=\rho H_{1}\left(T_{1} \cup T_{2}\right),
\end{aligned}
$$

which contradicts Lemma 5.

See the proof of [18, Theorem 2] for more details of the proof of Theorem 7.

Theorem 7. Suppose $X$ is a compact subset of the interior of a nonclosed, piecewise-linear 3-manifold $M^{3}$. Suppose each component of $X$ bas property $\mathrm{uv}\left(1, m ; Z_{p}\right)$ where $p$ is a prime or zero and $p=2$ if $M^{3}$ is nonorientable. Then there exists a compact polybedron $Z$ in Int $M^{3}$ such that each component of $Z$ is a 3-manifold, $X \subset$ Int $Z$, and there exists a $Z_{0}$ obtained from $Z$ by simple moves in $M^{3}$ such that each component $T$ of $\partial Z_{0}$ satisfies $\rho H_{1}\left(T ; Z_{p}\right)=\rho H_{1}\left(T ; Z_{2}\right) \leq 2 m$. 
Proof. We may suppose $M^{3}$ is compact. Assume $p$ is a prime. According to the Finiteness Theorem of Haken [7], [8], there exists a positive integer $H$ such that any collection of $H$ or more disjoint, incompressible, polyhedral surfaces in Int $M^{3}$ contains a pair of surfaces that are topologically parallel. Using an ANRsequence for $X$ in $M^{3}$ of the type described in the remark in $\$ 2$, we can find compact polyhedra $Z_{1}, \cdots, Z_{H+1}$ such that

(1) each component of $Z_{i}$ is a 3 -manifold, $i=1, \cdots, H+1$,

(2) $Z_{1} \subset$ Int $M^{3}$ and $Z_{i+1} \subset$ Int $Z_{i}, i=1, \cdots, H$,

(3) if $Z_{i+1}^{\prime}$ is a component of $Z_{i+1}$ and $Z_{i}^{\prime}$ is the component of $Z_{i}$ that contains $Z_{i+1}^{\prime}$ then $\rho$ image $\left[H_{1}\left(Z_{i+1}^{\prime} ; Z_{p}\right) \rightarrow H_{1}\left(Z_{i}^{\prime} ; Z_{p}\right)\right] \leq m, i=1, \cdots, H$, and

(4) $X \subset$ Int $Z_{H+1}$.

Call an $(H+1)$-tuple $\left\{Z_{1}, \cdots, Z_{H+1}\right\}$ admissible if it satisfies (1), (2), and (3). Lemma 4 shows that if $Z_{i}^{\#}$ is obtained from $Z_{i}$ by a simple annexation of type 2 or a simple reduction in $Z_{i-1}$ and $Z_{i+1} \subset$ Int $Z_{i}^{\#}$, then $\left\{Z_{1}, \cdots, Z_{i}^{\#}, \cdots, Z_{H+1}\right\}$ is admissible. (The exceptional cases $i=1$ or $H+1$ are left to the reader.) Hence there is an admissible $(H+1)$-tuple $\left\{Z_{1}^{*}, \cdots, Z_{H+1}^{*}\right\}$, obtained from $\left\{Z_{1}, \cdots, Z_{H+1}\right\}$ by simple annexations of type 2 or simple reductions, that admits no further such moves. Any boundary component of $Z_{i}^{*}$ that is not a 2 -sphere must be incompressible. (See the proof of $[18$, Theorem 2].)

If some $Z_{i}^{*}$ has only boundary components of rank $\leq 2 m$, that $Z_{i}^{*}$ is our required $Z_{0}$ for $Z=Z_{i}$. So suppose each $Z_{i}^{*}$ has a boundary component $T_{i}$ such that $\rho H_{1}\left(T_{i} ; Z_{p}\right)>2 m$. Now $T_{1}, \cdots, T_{H+1}$ are disjoint, incompressible, polyhedral surfaces in Int $M^{3}$ so some $T_{i}, T_{j}, i<j$, bound a parallelity component $A$ in $M^{3}$ (i.e., $A$ is piecewise-linearly homeomorphic to $T_{i} \times[0,1]$ and $\left.\partial A=T_{i} \cup T_{j}\right)$. By the Lemma in [7, Appendix] we can assume $j=i+1$ (we may need to replace $T_{k}$ by another boundary component of $\left.Z_{k}^{*}\right)$ and that Int $A \cap\left(\bigcup_{i=1}^{H+1} \partial Z_{i}^{*}\right)$ consists entirely of 2-spheres. Since $A$ is irreducible, $A$ is contained in $Z_{i}^{*}$ except for the interiors of finitely many disjoint 3-cells contained in Int $A$. Let $Z_{k}^{* \prime}$ be the component of $Z_{k}^{*}$ that contains $T_{k}, k=i$ or $i+1$. Hence

$$
\begin{aligned}
\rho \text { image }\left[H_{1}\left(Z_{i+1}^{* \prime} ; Z_{p}\right) \rightarrow H_{1}\left(Z_{i}^{* \prime} ; Z_{p}\right)\right] & \geq \rho \text { image }\left[H_{1}\left(T_{i+1} ; Z_{p}\right) \rightarrow H_{1}\left(Z_{i}^{* \prime} ; Z_{p}\right)\right] \\
& =\rho \text { image }\left[H_{1}\left(T_{i} ; Z_{p}\right) \rightarrow H_{1}\left(Z_{i}^{* \prime} ; Z_{p}\right)\right]>m .
\end{aligned}
$$

The last inequality follows from Corollary 6 . But now the admissibility of $\left\{Z_{1}^{*}, \ldots\right.$, $\left.Z_{H+1}^{*}\right\}$ is violated. The proof is complete for $p$ a prime once we show that if $T$ is an orientable surface, $\rho H_{1}\left(T ; Z_{p}\right)=\rho H_{1}\left(T ; Z_{2}\right)$. But $H_{1}(T ; Z)$ is a free abelian group of rank twice the genus of $T[15$, p. 132]. So by the universal coefficient theorem, $\rho H_{1}(T ; Z)=\rho H_{1}\left(T ; Z_{p}\right)=\rho H_{1}\left(T ; Z_{2}\right)$.

Suppose $p=0$. But uv( $1, m ; Z)$ implies uv $\left(1, m ; Z_{2}\right)$. Hence the above proof yields the desired conclusion. The proof of Theorem 7 is complete. 
Theorem 8. Let $M^{3}$ be a nonclosed piecewise-linear 3-manifold. Let $W_{i}$, $i=$ $1, \ldots, k$, be compact polybedra in Int $M^{3}$ such that each component of $W_{i}$ is a 3manifold and $W_{i+1} \subset$ Int $W_{i \cdot}$. Let $Z$ be a compact polybedron in Int $W_{k}$ such that each component $Q^{3}$ of $Z$ is a 3-manifold that has free image in some $W_{i}$ (i.e., there exists a component $R^{3}$ of $W_{i}$ such that $Q^{3} \subset R^{3}$ and image $\left[\pi_{1}\left(Q^{3}\right) \rightarrow \pi_{1}\left(R^{3}\right)\right]$ is a free group). Then by applying extended simple moves to $Z$ in $M^{3}$ we can obtain a $Z_{0}$ such that each component of $Z_{0}$ is simply connected.

Proof. Let $l$ be the smallest integer such that each component of $Z$ has free image in some $W_{s}, s \leq l$. We will induct on $l$.

Suppose $l=1$. Apply Theorem 1 of [20] to each component of $W_{1}$ with $\mathrm{P}$ being the property of being a trivial group.

Suppose $l>1$. There exists a $Z$, obtained from $Z$ by extended simple moves in $W_{l}$ and such that $Z_{1}$ admits no further extended simple moves in $W_{l}$. Let $Q_{1}^{3}$

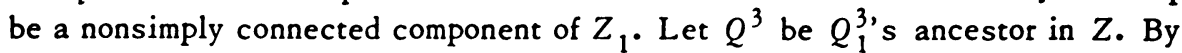
$\left[20\right.$, Lemma $\left.\mathrm{B}^{+}\right]$since $Q^{3}$ has free image in $W_{s}, s \leq l, Q_{1}^{3}$ has free image in $W_{s}$ But if $Q^{3}$ has free image in $W_{l}$ then Theorem 1 of [20] implies that $Q_{1}^{3}$ is simply connected. Hence each component of $Z_{1}$ has free image in some $W_{s}$ where $s \leq$ $l-1$. By inductive hypothesis there is a $Z_{0}$ obtained from $Z_{1}$ by extended simple moves in $M^{3}$ such that each component of $Z_{0}$ is simply connected. $Z_{0}$ is our required polyhedron.

Caution. Theorem 8 asserts only that there exists some way to obtain the required $Z_{0}$. Often there are $Z_{0}$ 's obtained from $Z$ by extended simple moves in $M^{3}$, that admit no further extended simple moves, and do not even have components with free fundamental group. This contrasts with Theorem 1 of [20] in which every $Z_{0}^{3}$ that admits no further extended simple moves satisfies the conclusion.

A piecewise-linear 3-manifold $P^{3}$ is irreducible if every polyhedral 2-sphere in Int $P^{3}$ bounds a 3 -cell. $P^{3}$ is prime if every polyhedral, separating 2-sphere in Int $P^{3}$ bounds a 3-cell. If $P_{1}^{3}$ and $P_{2}^{3}$ are piecewise-linear 3-manifolds the interior connected sum, denoted by $P_{1}^{3} \# P_{2}^{3}$, is obtained by choosing polyhedral 3-cells $B_{1}^{3}, B_{2}^{3}$ in Int $P_{1}^{3}$, Int $P_{2}^{3}$, respectively, removing Int $B_{1}^{3}$ and Int $B_{2}^{3}$, and identifying $\partial B_{1}^{3}$ and $\partial B_{2}^{3}$ by a piecewise-linear homeomorphism.

Lemma 9. If $R^{3}$ is a compact, irreducible, piecewise-linear 3-manifold that bas free fundamental group, then $R^{3}$ is a bandlebody or a bomotopy 3-sphere.

Proof. Suppose $R^{3}$ is closed. The only incompressible, 2-sided surfaces that $R^{3}$ can contain are 2-spheres. Theorem 1 of [27] shows $\pi_{1}\left(R^{3}\right)$ is trivial. Hence $R^{3}$ is a homotopy 3 -sphere.

Suppose $\partial R^{3} \neq \varnothing$ and $\partial R^{3}$ contains a surface other than a 2 -sphere. By the loop theorem [26], $R^{3}$ admits a simple reduction to $R_{1}$ (possibly not connected). 
Note each component of $R_{1}$ is irreducible and has free fundamental group. Hence there is a compact polyhedron $Z$ such that each component of $Z$ is a 3-cell and $Z$ is obtained from $R^{3}$ by a finite number of simple reductions. Since $R^{3}$ is obtained from $Z$ by adding a finite number of solid 1 -handles, $R^{3}$ is a handlebody.

Theorem 10. Suppose $R^{3}$ is a compact, piecewise-linear 3-manifold with free fundamental group. Then $R^{3}$ is piecewise-linearly homeomorphic to an interior connected sum of a finite number of bandlebodies, irreducible bomotopy 3-spheres, and $S^{2}$ bundles over $S^{1}$. ( $S^{n}$ denotes the $n$-sphere.)

Proof. The finiteness theorem [7], [8] implies that $R^{3}$ is piecewise-linearly homeomorphic to a finite interior connected sum $P_{1}^{3} \# \ldots \# P_{k}^{3}$ of prime 3-manifolds. The proof of Lemma 1 of [24] shows that any prime 3-manifold that is not irreducible is an $S^{2}$ bundle over $S^{1}$. Since the fundamental group of $R^{3}$ is isomorphic to the free product of the fundamental groups of $P_{1}^{3}, \ldots, P_{k}^{3}$, each $P_{i}^{3}$ has free fundamental group. By Lemma 9 each irreducible $P_{i}^{3}$ is a handlebody or a homotopy 3sphere.

4. 1-dimensional compact sets. In this section we investigate the relationship between arc pushing properties, separation properties, and definability by handlebodies for compact sets of dimension no larger than one.

Suppose $\epsilon>0$. A handlebody $H^{3}$ is $\epsilon-t$ thin if there exists a collection $B_{1}, \cdots$, $B_{l}$ of polyhedral 3-cells such that $\bigcup_{i=1}^{l} B_{i}=H^{3}, B_{i} \cap B_{j}$ is either empty or a polyhedral 2-cell $D_{i, j}$ for $i \neq j, B_{i} \cap B_{j} \cap B_{k}=\varnothing$ for $i, j$, and $k$ distinct, and each $B_{i}$ has diameter less than $\epsilon$.

Remarks. (1) The union of any subcollection of $B_{1}, \cdots, B_{l}$ is a collection of $\epsilon$-thin handlebodies. (2) If $T$ is a triangulation of a 3-manifold of mesh less than $\epsilon$ and $L$ is a 1 -dimensional subcomplex of $T$, then a second derived regular neighborhood of $L$ yields a $2 \epsilon$-handlebody. The required $B_{i}$ correspond to the stars of the vertices of the induced first derived subdivision of $L$. (3) Suppose $G$ is a polyhedral, finite graph in Int $H^{3}$ such that $G \cap D_{i, j}$ is exactly one point for all $D_{i, j}$ 's and we can consider $B_{i}$ to be a piecewise-linear cone over $\partial B_{i}$ such that $G \cap B_{i}$ corresponds to a piecewise-linear subcone over $G \cap \partial B_{i}$. Then $H^{3} \epsilon$-retracts onto $G$ by a retraction that maps $B_{i}$ into $B_{i}$ for all $i$. (4) Suppose $H^{3}$ is a subpolyhedron of the interior of a 3-manifold $M^{3}$ and $G$ is a graph as described in Remark (3). Suppose $N$ is a regular neighborhood of $G$ in Int $H^{3}$ such that $N \cap$ $D_{i, j}$ is a 2 -cell for all $D_{i, j}$. Let $U$ be a neighborhood of $H^{3}$. Then there is a piecewise-linear $\epsilon$-homeomorphism (i.e., moves no point as much as $\epsilon$ ) $f$ of $M^{3}$ onto itself such that $f(N)=H^{3}, f\left(N \cap D_{i, j}\right)=D_{i, j}$, and $f$ is the identity off $U$.

Suppose $X$ is a proper, compact subset of the interior of a piecewise-linear 3-manifold $M^{3}$. (Keep this notation for the remainder of the paragraph.) $X$ is 
definable by $\epsilon$-thin bandlebodies if $X$ is definable by 3 -manifolds each component of which is an $\epsilon$-thin handlebody. $X$ is definable by thin bandlebodies if, for each $\epsilon>0, X$ is definable by $\epsilon$-thin handlebodies. $X$ has the (strong) arc pusbing property if, given $\epsilon>0$ and a polyhedral arc $A$ contained in Int $M^{3}$ such that $X \cap \partial A$ $=\varnothing$, there is a piecewise-linear $(\epsilon)$-homeomorphism $b$ of $M^{3}$ onto $M^{3}$ such that $b$ is the identity outside the $\epsilon$-neighborhood of $X$ and $b(A) \cap X=\varnothing$. (McMillan [16] has shown in orientable 3-manifolds the arc pushing property is equivalent to being definable by handlebodies. No essential change in his proof is needed to drop orientability. The arc pushing properties have also been investigated in [4], [5], [23]).

The proof of Lemma 11 is immediate from Remark (1).

Lemma 11. Suppose $X$ is a compact, proper subset of the interior of a piecewise-linear 3-manifold. Suppose $Y$ is a closed subset of $X$. If $X$ is definable by thin bandlebodies, then $Y$ is definable by thin bandlebodies.

Lemma 12. Suppose $X$ is a compact metric space. $X$ bas dimension less than or equal to one if and only if there is an embedding of $X$ in $E^{3}$ that is definable by thin bandlebodies.

Proof. If $\operatorname{dim}(X) \leq 1$, then $X$ embeds in $M$, Menger's universal 1-dimensional curve [11]. Lefschetz [11, p. 529] embeds $M$ in $E^{3}$ so that $M$ is definable by thin handlebodies. (See Remark (2).) Hence by Lemma $11, X$ embeds in $E^{3}$ so as to be definable by thin handlebodies.

Using Remark (3), we see that if $X$ is definable by $\epsilon$-thin handlebodies, $X \epsilon$ maps onto a finite graph. Hence $X$ is at most 1-dimensional.

Theorem 13. Suppose $X$ is a compact, proper subset of the interior of a piecewise-linear 3-manifold $M^{3}$. The following statements are equivalent.

A. $X$ is definable by thin handlebodies.

B. If $p$ and $q$ are distinct points in $X$ there is a tame (i.e., definable by 3cells in $M^{3}$ ), compact, 0-dimensional subset of $X$ that separates $p$ and $q$.

C. $X$ bas the strong arc pusbing property.

Proof. We can assume $M^{3}$ is compact. In order to show statement A implies statement $\mathrm{B}$, let $p$ and $q$ be distinct points of $X$. It is sufficient to find a tame 2-sphere in Int $M^{3}$ that separates $p$ from $q$ and intersects $X$ in a 0 -dimensional set. Let $S$ be a polyhedral 2-sphere in Int $M^{3}$ that separates $p$ and $q$. There is a $\delta>0$ such that if $b$ is a $\delta$-homeomorphism of $M^{3}$ onto $M^{3}$, then $b(S)$ separates $p$ and $q$. In view of the method for constructing homeomorphisms given in [2, Theorem 7.1], it is sufficient to show that given $\epsilon>0$ and a polyhedral 2-sphere $S_{1}$ in Int $M^{3}$ there is a piecewise-linear $\epsilon$-homeomorphism $f$ of $M^{3}$ onto itself such that $f\left(s_{1}\right) \cap X$ has components of diameter less than $\epsilon$. Suppose $\epsilon>0$ and a 
polyhedral 2-sphere $S_{1}$ in Int $M^{3}$ are given. There exists a disjoint collection $H_{1}^{3}, \cdots, H_{r}^{3}$ of $\epsilon$-thin handlebodies in Int $M^{3}$ whose union contains $X$. Let $U_{1}$, $\cdots, U$, be disjoint neighborhoods of $H_{1}^{3}, \cdots, H_{r}^{3}$, respectively. Let $H^{3}$ be one of $H_{1}^{3}, \cdots, H_{r}^{3}$ and $U$ be the corresponding $U_{1}, \cdots, U_{r}$. Recall the notation used in defining $H^{3}$ as an $\epsilon$-thin handlebody. We can assume that no $D_{i, j}$ is entirely contained in $S_{1}$. Hence we can pick a graph $G$, as in Remark (3), such that, for all $D_{i, j}, G \cap S_{1} \cap D_{i, j}=\varnothing$. Now select a regular neighborhood $N$ of $G$ in $H^{3}$, as in Remark (4), such that $N \cap S_{1} \cap D_{i, j}=\varnothing$ for all $D_{i, j}^{\prime}$ 's. Hence there exists a piecewise-linear $\epsilon$-homeomorphism $g$ of $M^{3}$ onto itself such that $g$ is fixed off $U, g(N)=H^{3}$, and $g\left(N \cap D_{i, j}\right)=D_{i, j}$. Therefore each component of $g\left(S_{1}\right) \cap H^{3}$ is contained in some $B_{i}$ and hence has diameter less than $\epsilon$. Let $g_{1}, \cdots, g_{r}$ be the homeomorphisms obtained for $H_{1}^{3}, \cdots, H_{r}^{3}$, respectively. Let $f$ be the composition of $g_{1}, \cdots, g_{r}$. Each component of $f\left(S_{1}\right) \cap\left(\bigcup_{i=1}^{r} H_{i}^{3}\right)$ has diameter less than $\epsilon$. So each component of $f\left(S_{1}\right) \cap X$ has diameter less than $\epsilon$. Hence $f$ is our required homeomorphism.

We will now show statement B implies statement C. Assume statement B holds. We can strengthen statement $B$ by the following observations. The alternative proof of Theorem 6.1 of [2] and Theorem II 2 of [10] show a finite union of tame, 0 -dimensional, compact subsets of Int $M^{3}$ is a tame, 0 -dimensional compact subset. By simple compactness arguments we can establish the following two statements in order. If $p \in X$ and $Y$ is a closed subset of $X$ that misses $p$ then there is a tame 0 -dimensional compact subset $P$ of $X$ that separates $p$ from all points of $Y-P$. If $\epsilon>0$ there exists a tame, 0 -dimensional, compact subset $P_{\epsilon}$ of $X$ such that each component of $X-P_{\epsilon}$ has diameter less than $\epsilon$.

Suppose $\epsilon>0$ and $A$ is a polygonal arc in Int $M^{3}$ such that $X \cap \partial A=\varnothing$. Since $X$ contains no open subset of $M^{3}$, there is a piecewise-linear $\epsilon / 3$-homeomorphism $b$ of $M^{3}$ onto itself such that $b$ is fixed off the $\epsilon / 3$-neighborhood of $X$ and each component of $b(A) \cap X$ has diameter less than $\epsilon / 3$. Let $A_{1}, \cdots, A_{t}$ be disjoint subarcs of $b(A)$ of diameter less than $\epsilon / 3$ such that $A_{i} \cap X \neq \varnothing$ and $X \subset$ $\bigcup_{i=1}^{t}$ Int $A_{i}$. Let $U_{1}, \cdots, U_{t}$ be disjoint neighborhoods of $A_{1}, \cdots, A_{t}$, respectively, of diameter less than $\epsilon / 3$. In the remainder of this proof our homeomorphisms will be fixed off $\bigcup_{i=1}^{t} U_{i}$. Note $\bigcup_{i=1}^{t} U_{i}$ is contained in the $\epsilon$-neighborhood of $X$. Let $D_{1}, \cdots, D_{t}$ be polyhedral 2-cells such that $D_{i} \subset U_{i}, D_{i} \cap b(A)=A_{i}$ and $A_{i} \cap \partial D_{i}=\partial A_{i}$. Since $\partial A_{i}$ misses $X$ we can find disjoint subarcs $A_{i}^{\prime}, A_{i}^{\prime \prime}$ of $\partial D_{i}$ such that $A_{i}^{\prime}, A_{i}^{\prime \prime}$ miss $X$ and $A_{i}^{\prime}, A_{i}^{\prime \prime}$ each contain one endpoint of $A_{i}$. Let $E_{i}^{\prime}, E_{i}^{\prime \prime}$ be the closures of the two components of $\partial D_{i}-\left(A_{i}^{\prime} \cup A_{i}^{\prime \prime}\right)$. Let $\delta$ be the minimum of the distance from $E_{i}^{\prime}$ to $E_{i}^{\prime \prime}, i=1, \cdots, t$; the distance from $X$ to $A_{i}^{\prime} \cup A_{i}^{\prime \prime}, i=1, \cdots, t$; the distance from $X$ to the closure of $b(A)-\bigcup_{i=1}^{t} A_{i}$. There exists a piecewise-linear $\delta / 3$-homeomorphism $f$ of $M^{3}$ onto $M^{3}$ such that $f$ is fixed off 


$$
\bigcup_{i=1}^{t} U_{i}, f\left(b(A) \cup\left(\bigcup_{i=1}^{t} D_{i}\right)\right) \cap X \subset \bigcup_{i=1}^{t} f\left(D_{i}-\left(A_{i}^{\prime} \cup A_{i}^{\prime \prime}\right)\right),
$$

and $f\left(\bigcup_{i=1}^{t} D_{i}\right)$ misses $P_{\delta / 3^{\prime}} \cdot\left(P_{\delta / 3}\right.$ is defined in the preceding paragraph.) Therefore each component of $f\left(D_{i}\right) \cap X$ has diameter less than $\delta / 3$. Note no component of $f\left(D_{i}\right) \cap X$ separates $f\left(A_{i}^{\prime}\right)$ from $f\left(A_{i}^{\prime \prime}\right)$ in $f\left(D_{i}\right)$ since $f\left(E_{i}^{\prime}\right)$ and $f\left(E_{i}^{\prime \prime}\right)$ are at least a distance $\delta / 3$ apart. In $f\left(D_{i}\right)$ select a polyhedral arc $A_{i}^{*}$ such that $A_{i}^{*} \cap f\left(\partial D_{i}\right)=f\left(\partial A_{i}\right)$ and $A_{i}^{*}$ misses $X$. There is a piecewise-linear $\epsilon / 3$-homeomorphism $g$ such that $g$ is fixed off $\bigcup_{i=1}^{t} U_{i}, g f\left(A_{i}\right)=A_{i}^{*}$ and $g$ is fixed on $f\left(b(A)-\left(\bigcup_{i=1}^{t}\right.\right.$ Int $\left.\left.A_{i}\right)\right)$. Now $g f b$ is a piecewise-linear $\epsilon$-homeomorphism fixed off the $\epsilon$-neighborhood of $X$ such that $g f b(A) \cap X=\varnothing$.

In conclusion we will show statement $C$ implies statement $A$. Let $U$ be a neighborhood of $X$ in $M^{3}$ and suppose $\epsilon>0$. Let $W$ be a compact polyhedron in $U$ such that each component of $W$ is a 3 -manifold and $X \subset$ Int $W$. Suppose $T$ is a triangulation of $W$ such that the mesh of $T$ is less than $\epsilon / 18$ and $T^{\prime \prime}$. is a second derived subdivision of $T$ such that no new vertex of $T^{\prime}$ lies in $X$. ( $X$ contains no open set.) Let $T^{(1)}$ be the 1-skeleton of $T$ and $T_{(1)}$ be the dual 1-skeleton (i.e., the collection of all 1-simplexes and vertices of $T^{\prime}$ that miss $\left.T^{(1)}\right)$. Let $H^{1}, H_{1}$ be the simplicial neighborhoods of $T^{(1)}, T_{(1)}$, respectively, in $T^{\prime \prime}$. Then $H^{1} \cup H_{1}$ $=W, H^{1} \cap H_{1} \subset \partial H^{1} \cap \partial H_{1}$ and $H^{1}, H_{1}$ are collections of $\epsilon / 9$-thin handlebodies. (See Remark (2).) Let $A_{1}, \cdots, A_{s}$ be the 1-simplexes of $T_{(1)}$. The endpoints of $A_{1}, \cdots, A_{s}$ miss $X$. Apply the strong arc pushing property to inductively choose $\epsilon_{i}, b_{i}, 1 \leq i \leq s$; such that $\epsilon_{i}$ is less than $\epsilon / 9 s, \epsilon_{i}$ is less than the distance from $X$ to $b_{i-1} \cdots b_{1}\left(A_{1} \cup \cdots \cup A_{i-1}\right), \epsilon_{i}$ is less than the distance from $X$ to $\partial A_{i+1}$ $\cup \cdots \cup \partial A_{s}, \epsilon_{i}$ is less than the distance from $X$ to $b_{i-1} \cdots b_{1}(\partial W)=\partial W$, and $b_{i}$ is a piecewise-linear $\epsilon_{i}$-homeomorphism of $M^{3}$ onto itself such that $b_{i} b_{i-1} \cdots b_{1}\left(A_{i}\right)$ $\cap X=\varnothing$. Let $b=b_{s} \cdots b_{1}$. Then $b$ is a piecewise-linear $\epsilon / 9$-homeomorphism of $M^{3}$ onto $M^{3}$ such that $b(W)=W$ and $b\left(T_{(1)}\right) \cap X=\varnothing$. Note $b\left(H^{1}\right), b\left(H_{1}\right)$ are collections of $\epsilon / 3$-thin handlebodies. Let $N$ be a nice regular neighborhood of $b\left(T_{(1)}\right)$ in $b\left(H_{1}\right)$ that misses $X$. There is a piecewise-linear $\epsilon / 3$-homeomorphism $f$ of $M^{3}$ onto $M^{3}$ such that $f b\left(H_{1}\right)$ misses $X, f b($ Int $W)$ contains $X$ and $f b(W) \subset U$. (The inverse of the type of homeomorphism promised in Remark (4) is almost our f. The situation is slightly changed since $T_{(1)}$ intersects $\left.\partial H_{1}.\right)$ Hence $f b\left(H^{1}\right)$ is a collection of $\epsilon$-thin handlebodies contained in $U$ that contain $X$. The proof of Theorem 13 is complete.

Corollary 14. There is an embedding $b$ of Menger's universal 1-dimensional curve $M$ in $E^{3}$ and distinct points $p$ and $q$ in $b(M)$ such that (1) every 0-dimensional compact subset of $h(M)$ that separates $p$ and $q$ is wild, and (2) every tame surface in $E^{3}$ that separates $p$ and $q$ must intersect $b(M)$ in a 1-dimensional set. 
Proof. The embeddings of $M$ given in [4], [5], [23] all suffice in view of Theorem 13. Bothe [5] even gives an embedding definable by handlebodies.

5. Main theorem and corollaries. Our main result is the following theorem.

Theorem 15. Suppose $m$ and $l$ are nonnegative integers and that $p$ is either zero or a prime. Let $Y$ be a compact metric space such that each component of $Y$ bas properties uv $\left(1, m ; Z_{p}\right), \mathrm{uv}\left(2, l ; Z_{p}\right)$ and UVF. Suppose $X$ is a compact metric space and $\operatorname{Sh}(Y) \geq \operatorname{Sh}(X)$.

Let $b$ be a topological embedding of $X$ into the interior of a nonclosed, piecewiselinear 3-manifold $M^{3}$. If $M^{3}$ is not orientable assume $p=2$. Then $b(X)=\bigcap_{i=1}^{\infty} H_{i}$ where $H_{i}$ is a compact polybedron in Int $M^{3}$ such that each component of $H_{i}$ is a 3-manifold with free fundamental group and at most $l+1$ boundary components, and $H_{i+1} \subset$ Int $H_{i}$.

Proof. We can assume $M^{3}$ is compact. Suppose $b(X) \subset U \subset M^{3}$ where $U$ is an open set. It is sufficient to find a compact polyhedron $H \subset U$ such that each component of $H$ is a 3 -manifold with free fundamental group and at most $l+1$ boundary components, and such that $b(X) \subset$ Int $H$. Note that Theorem 1 and Lemma 2 imply that $b(X)$ has properties $u v\left(1, m ; Z_{p}\right), u v\left(2, l ; Z_{p}\right)$, and UVF.

Recalling the remark in $\$ 2$ and Theorem 3 we can find compact polyhedra $W_{0}$, $\cdots, W_{k}$, whose components are 3 -manifolds, such that

(1) $W_{i+1} \subset$ Int $W_{i}, i=0, \cdots, k-1, X \subset$ Int $W_{k}, W_{0} \subset U$,

(2) each component of $W_{k}$ has at most $l+1$ boundary components,

(3) for each component $W_{i+1}^{\prime}$ of $W_{i+1}$ the inclusion induced homomorphism $\pi_{1}\left(W_{i+1}^{\prime}\right) \rightarrow \pi_{1}\left(W_{i}^{\prime}\right)$ factors through a free group, $i=0, \ldots, k-1\left(W_{i}^{\prime}\right.$ is the component of $W_{i}$ that contains $\left.W_{i+1}^{\prime}\right)$, and

(4) $k>\left(\rho H_{2}\left(M^{3} ; Z_{2}\right)+l+1\right) 2 m+\rho H_{1}\left(M^{3} ; Z_{2}\right)+1$

By Theorem 7 we can find compact polyhedra $Z$ and $Z_{0}$ such that each component of $Z$ is a 3-manifold, $b(X) \subset Z \subset$ Int $W_{k}, Z_{0}$ is obtained from $Z$ by simple moves in $W_{k}$, and every component $T$ of $\partial Z_{0}$ satisfies $\rho H_{1}\left(T ; Z_{p}\right)=\rho H_{1}\left(T ; Z_{2}\right)$ $\leq 2 m$. Let $L_{1}, \cdots, L_{s}$ be the components of $W_{k}-$ Int $Z_{0}$ that do not intersect $\partial W_{k}$. Let $V=Z_{0} \cup L_{1} \cup \ldots \cup L_{s} \subset$ Int $W_{k}$. Note $\partial V \subset \partial Z_{0}$.

Our goal in the next few paragraphs is to show each component of $V$ has free image in some $W_{i}$. Let $Q^{3}$ be a component of $V$ and $W_{k}^{\prime}$ be the component of $W_{k}$ that contains $Q^{3}$. Since $W_{k}^{\prime}$ has at most $l+1$ boundary components, $W_{k}^{\prime}-\operatorname{Int} Q^{3}$ has at most $l+1$ components. Hence $M^{3}-$ Int $Q^{3}$ has at most $l+1$ components.

We will now show $Q^{3}$ has at most $\rho H_{2}\left(M^{3} ; Z_{2}\right)+l+1$ boundary components. Suppose $s_{1}, \cdots, s_{t}$ are the boundary components of $Q^{3}, M^{3}-$ Int $Q^{3}$ has $r \leq l+1$ components, and $s_{1}, \cdots, s_{t}$ are numbered so that $s_{t-r+1}, \cdots, s_{t}$ lie in different components of $M^{3}-$ Int $Q^{3}$. Since $M^{3}-\left(s_{1} \cup \ldots \cup S_{t-r}^{t-r+1}\right)$ is connected, $s_{1}, \ldots$, 
$S_{t-r}$ are carriers for linearly independent elements of $H_{2}\left(M^{3} ; Z_{2}\right)$. So $t-r \leq$ $\rho H_{2}\left(M^{3} ; Z_{2}\right)$ and $t \leq \rho H_{2}\left(M^{3} ; Z_{2}\right)+l+1$ as asserted. Hence $\rho H_{1}\left(\partial Q^{3} ; Z_{2}\right) \leq$ $\left(\rho H_{2}\left(M^{3} ; Z_{2}\right)+l+1\right) 2 m$. Using the Mayer-Vietoris sequence for $Q^{3}$ and the closure of $M^{3}-Q^{3}$ with coefficients $Z_{2}$, we see that image $\left[H_{1}\left(\partial Q^{3} ; Z_{2}\right) \rightarrow H_{1}\left(Q^{3}, Z_{2}\right)\right]$ contains $\operatorname{ker}\left[H_{1}\left(Q^{3} ; Z_{2}\right) \rightarrow H_{1}\left(M^{3} ; Z_{2}\right)\right]$. Hence

$$
\begin{aligned}
\rho H_{1}\left(Q^{3} ; Z_{2}\right) \leq & \rho \text { image }\left[H_{1}\left(Q^{3} ; Z_{2}\right) \rightarrow H_{1}\left(M^{3} ; Z_{2}\right)\right] \\
& +\rho \operatorname{ker}\left[H_{1}\left(Q^{3} ; Z_{2}\right) \rightarrow H_{1}\left(M^{3} ; Z_{2}\right)\right] \\
& \leq \rho H_{1}\left(M^{3} ; Z_{2}\right)+\rho H_{1}\left(\partial Q^{3} ; Z_{2}\right)<K-1 .
\end{aligned}
$$

We will use primes to denote components. There are components $W_{0}^{1}, \cdots$, $W_{k}^{\prime}$ of $W_{0}, \cdots, W_{k}$ such that $Q^{3} \subset W_{k}^{\prime} \subset \cdots \subset W_{0}^{\prime}$. Recall that $\pi_{1}\left(W_{i}^{\prime}\right) \rightarrow \pi_{1}\left(W_{i-1}^{\prime}\right)$ factors through a free group $F_{i}$. Let $f_{i}: \pi_{1}\left(W_{i}^{\prime}\right) \rightarrow F_{i}$ and $g_{i}: F_{i} \rightarrow \pi_{1}\left(W_{i-1}^{\prime}\right)$ be factoring homomorphisms. The following diagram is consistent. We define $b_{i}=$ $f_{i-1} g_{i}: F_{i} \rightarrow F_{i-1}$.

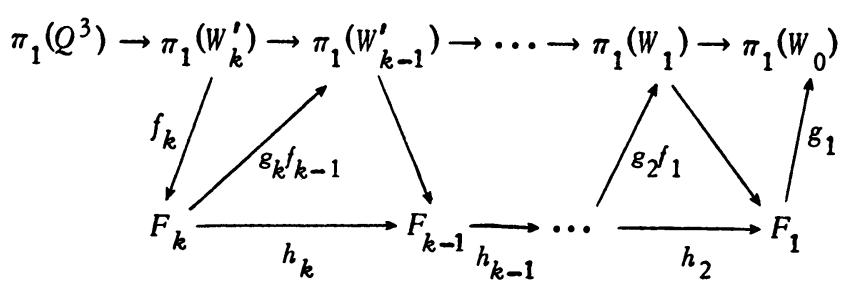

Let $I_{i}=$ image $\left[\pi_{1}\left(Q^{3}\right) \rightarrow F_{i}\right], i=1, \cdots, k$. Let $\theta_{i}=\left.h_{i}\right|_{I_{i}}$. Note that $\theta_{i}$, $i=2, \cdots, k$, is an epimorphism. Hence $\rho I_{k} \geq \rho I_{k-1} \geq \cdots \geq \rho I_{1} \geq 0$. Consider the consistent diagram

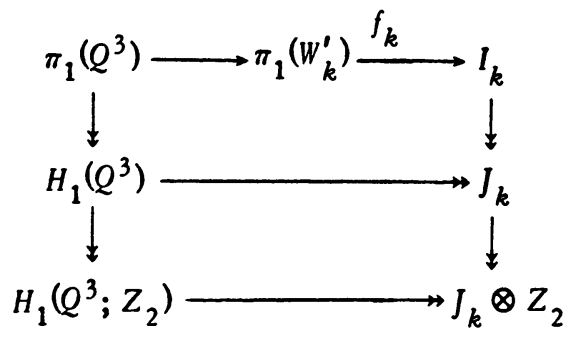

where the top vertical arrows represent abelianization epimorphisms, $J_{k}$ is the abelianization of $l_{k}$, the middle horizontal arrow is the epimorphism induced by the top half of the diagram, the bottom vertical arrows are tensor product epimorphisms, and the bottom horizontal arrow is the epimorphism induced from the diagram. Since $I_{k}$ is a free group, $\rho I_{k}=\rho J_{k}=\rho J_{k} \otimes Z_{2}$. Therefore, $\rho I_{k} \leq \rho H_{1}\left(Q^{3} ; Z_{2}\right)<k-1$. It follows that, for some $i, I_{i}$ and $I_{i-1}$ are free groups 
on the same number of generators. So by $[12, \mathrm{p} .312] \theta_{i}$ is an isomorphism, $\left.g_{i}\right|_{I_{i}}$ is a monomorphism, and so $Q^{3}$ has free image in $W_{i-1}^{\prime}$.

Now we can apply Theorem 8 to find a $V_{0}$ obtained from $V$ by extended simple moves in $U$ such that each component of $V_{0}$ has free fundamental group. Recall that $h(X) \subset Z \subset U, Z_{0}$ is obtained from $Z$ by simple moves in $U$, and $Z_{0} \subset V$. By

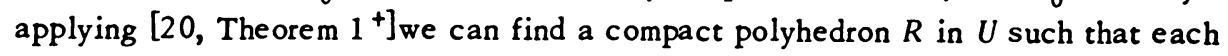
component of $R$ is a 3-manifold with free fundamental group and $b(X) \subset$ Int $R$. Hence $h(X)$ is definable by free 3 -manifolds.

The $R$ we obtained in the preceding paragraph satisfies the conditions we require for $H$ except possibly some component of $R$ may have more than $l+1$ boundary components. Keep the notation of the preceding paragraphs. Let $P$ be a compact polyhedron in Int $R$ such that each component $P^{\prime}$ of $P$ is a 3-manifold with free fundamental group, $h(X) \subset$ Int $P$, and $\rho$ image $\left[H_{2}\left(P^{\prime} ; Z_{p}\right) \rightarrow\right.$ $\left.H_{2}\left(R^{\prime} ; Z_{p}\right)\right] \leq l$ where $R^{\prime}$ is the component of $R$ that contains $P^{\prime}$.

Using Theorem 10 we see that $P^{\prime}$ is homeomorphic to $P_{1} \# \cdots \# P_{\text {, where }}$ each $P_{i}$ is either a homotopy 3-sphere, $S^{2}$ bundle over $S^{1}$, or a handlebody. Let $T_{1}, \cdots, T_{r-1}$ be dis joint, polyhedral 2-spheres in $P^{\prime}$ such that the components of $P^{\prime}$ cut along $T_{1}, \cdots, T_{r-1}$ are equivalent, in the sense of Milnor [24], to $P_{1}, \cdots, P_{r}$. Note that if $J$ is a boundary component of $P^{\prime}$, then $J$ plus some subcollection of $T_{1}, \cdots, T_{r-1}$ bounds a compact 3 -manifold $N_{J} \subset P^{\prime} .\left(N_{J}\right.$ is homeomorphic to a handlebody minus the interiors of a disjoint collection of 3-cells.)

Let $C$ be a small regular neighborhood of $T_{1} \cup \cdots \cup T_{r-1}$ in $P^{\prime}$. Let $U_{1}, \cdots, U_{p}$ be the components of $R^{\prime}-$ Int $C$ and $V_{1}, \cdots, V_{q}$ be the components of $P^{\prime}-$ Int $C$. Note $\partial U_{i} \cap \operatorname{Int} R^{\prime}$ and $\partial V_{j} \cap$ Int $P^{\prime}$ consist of 2-spheres. Hence each $U_{i}$ and $V_{j}$ has free fundamental group. Let $D$ be $C$ union the $U_{i}$ 's that miss $\partial R^{\prime}$. Each component of $D$ has free fundamental group and 2-sphere boundary components. Let $H^{\prime}$ be $D$ union the $V_{j}$ 's that miss Int $D$. Note $H^{\prime}$ is connected, $\pi_{1}\left(H^{\prime}\right)$ is free, $\partial H^{\prime} \subset \partial P^{\prime}$, and $H^{\prime} \supset P^{\prime}$. If $N$ is a component of $R^{\prime}-$ Int $H^{\prime}$ that misses $\partial R^{\prime}$, let $J_{1}, \cdots, J_{t}$ be the components of $N \cap H^{\prime}$. Then $N \cup N_{J_{1}} \cup \cdots$ $\cup N_{J_{t}}$ contains some $U_{i}$ that should have been added to $C$ to obtain $D$. Hence each component of $R^{\prime}$ - Int $H^{\prime}$ must intersect $\partial R^{\prime}$. Note also that if $P^{\prime \prime}$ is another component of $P$ then $H^{\prime}$ contains $P^{\prime \prime}$ or $H^{\prime} \cap P^{\prime \prime}=\varnothing$. Now the last three paragraphs of the proof of Theorem 3 apply to give us our required $H$. The proof of Theorem 15 is complete.

A (homotopy cube-with-handles) homotopy handlebody is a 3-manifold homeomorphic to the interior connected sum of a homotopy 3-sphere and a (cube-withhandles) handlebody. The meaning of being definable by homotopy handlebodies (or homotopy cubes-with-handles) should be clear.

If $X$ is a compact metric space Borsuk [3] says the fundamental dimension 
of $X$ is less than or equal to one, written $\mathrm{Fd}(X) \leq 1$, if there exists an at most 1dimensional compact metric space $Y$ such that $\operatorname{Sh}(Y) \geq \operatorname{Sh}(X)$.

Corollary 16. Suppose $m$ is a nonnegative integer and $p=0$ or is a prime. Suppose $X$ is a compact metric space such that each component of $X$ has properties uv $\left(1, m ; Z_{p}\right)$, uv $\left(2,0 ; Z_{p}\right)$ and UVF. Suppose $h$ is a topological embedding of $X$ into the interior of a nonclosed, piecewise-linear 3-manifold $M^{3}$. If $M^{3}$ is nonorientable assume $p=2$. Then $(1) h(X)$ is definable by homotopy handlebodies and (2) If $M^{3}$ is orientable, $h(X)$ is definable by homotopy cubes-with-handles.

Proof. (2) follows from (1). By Theorem 15 we know that $h(X)$ is definable by free 3-manifolds each component of which has connected boundary. Let $U$ be a neighborhood of $h(X)$ in $M^{3}$ such that $\rho$ image $\left[H_{2}\left(U ; Z_{p}\right) \rightarrow H_{2}\left(M^{3} ; Z_{p}\right)\right]=0$. It is sufficient to show that any compact piecewise-linear 3-manifold contained in $U$ with free fundamental group and connected boundary must be a homotopy handlebody. Suppose $W^{3}$ is a compact, piecewise-linear 3-manifold in $U$ with $\pi_{1}\left(W^{3}\right)$ free and $\partial W^{3}$ connected. By Theorem 10, if $W^{3}$ is not a homotopy handlebody then $W^{3}$ contains a nonseparating, polyhedral 2-sphere $S$. But $S \subset U$ and $S$ is the carrier of a representative of a nontrivial element of $\mathrm{H}_{2}\left(M^{3} ; Z_{p}\right)$. Our choice of $U$ makes this impossible. Hence $W^{3}$ must be a homotopy handlebody.

Corollary 17. Let $m$ be a nonnegative integer and let $p=0$ or a prime. Suppose $X$ is a compact metric space such that each component of $X$ has property $\mathrm{uv}\left(1, m ; Z_{p}\right)$. Let $h$ be a topological embedding of $X$ into the interior of $a$ nonclosed, piecewise-linear 3-manifold $M^{3}$. If $M^{3}$ is nonorientable let $p=2$. Then (1) if the dimension of $X$ is $\leq 1, h(X)$ is definable by handlebodies, and (2) if $\mathrm{Fd}(X) \leq 1$, then $h(X)$ is definable by homotopy handlebodies.

Proof. If the dimension of $X$ is $\leq 1$, by Lemma 12 we can embed $X$ in $E^{3}$ so as to be definable by thin handlebodies. Hence $X$ has properties uv $\left(2,0 ; Z_{p}\right)$ and UVF. By Corollary $16, h(X)$ is definable by homotopy handlebodies. Using Theorem 4 of [23] we see that we can choose the homotopy handlebodies to be handlebodies.

If $\mathrm{Fd}(X) \leq 1$ there exists a compact metric space $Y$ such that $\operatorname{dim}(Y) \leq 1$ and $\operatorname{Sh}(Y) \geq \operatorname{Sh}(X)$. By Lemma 12, $Y$ has properties UVF and uv $\left(2,0 ; Z_{p}\right)$. Hence by Theorem 1, $X$ has the same properties. Using Corollary 16 we see that $h(X)$ is definable by homotopy handlebodies. The proof is complete.

A continuum is a compact connected metric space. A topological space is locally $n$-connected, $n \geq 0$, if the space is locally connected in dimension $i$ for $0 \leq i \leq n$. See [9].

Lemma 18. Let $p=0$ or a prime and let $n$ be a nonnegative integer. Suppose 
$X$ is a locally n-connected continuum contained in the interior of a nonclosed, piecewise-linear 3-manifold $M^{3}$. Then there exists a compact, polyhedral 3 manifold $N^{3}$ such that

$$
X \subset \text { Int } N^{3}, \quad N^{3} \subset \text { Int } M^{3},
$$

$$
\begin{aligned}
\operatorname{image}\left[H_{i}\left(X ; Z_{p}\right) \rightarrow H_{i}\left(M^{3} ; Z_{p}\right)\right]=\operatorname{image}\left[H_{i}\left(N^{3} ; Z_{p}\right) \rightarrow H_{i}\left(M^{3} ; Z_{p}\right)\right] \\
\\
\text { for } 0 \leq i \leq n+1,
\end{aligned}
$$

and

$$
\text { image }\left[\pi_{1}(X) \rightarrow \pi_{1}\left(M^{3}\right)\right]=\text { image }\left[\pi_{1}\left(N^{3}\right) \rightarrow \pi_{1}\left(M^{3}\right)\right]
$$

Proof. We may assume $M^{3}$ is compact. Since $M^{3}$ is an ANR there is an $\epsilon>0$ such that any two $\epsilon-c$ lose maps from a compact metric space into $M^{3}$ are homotopic. There exists a $\delta>0$ such that if $N^{3}$ is a compact polyhedral 3 . manifold in the $\delta$-neighborhood of $X$ and $\sigma=\Sigma n_{j} \sigma_{j}$ is a $\delta$-small $i$-cycle in $N^{3}$ (i.e., each singular $i$-simplex $\sigma_{j}$ has diameter less than $\delta, \sigma_{j} \subset N^{3}, n_{j}$ belongs to $Z_{p}$, and $\sigma$ represents an element of $\left.H_{i}\left(N^{3} ; Z_{p}\right)\right)$ there is a singular $i$-cycle $\sigma^{\prime}=$ $\Sigma_{n_{j}} \sigma_{j}^{\prime}$ in $X$ such that $\sigma_{j}$ and $\sigma_{j}^{\prime}$ are $\epsilon$-close for each $j$. (See Theorem 4.1 of Chapter 5 of [9].) Hence $\sigma$ and $\sigma^{\prime}$ represent the same element of $H_{i}\left(M^{3} ; Z_{p}\right)$. We leave the proof of the condition on images of fundamental groups to the reader.

Addendum. If, for some $i \leq n+1, H_{i}\left(X ; Z_{p}\right)$ is finitely generated, then $X$ has property uv $\left(i, m ; Z_{p}\right)$ where $m=\rho H_{i}\left(X ; Z_{p}\right)$.

The last two corollaries of Theorem 15 are stated only in terms of a continuum. Of course there are similar corollaries where we apply the hypotheses to the components of a compact set.

Corollary 19. Suppose $X$ is a compact ANR and $b$ is a topological embedding of $X$ into the interior of a nonclosed, piecewise-linear 3-manifold $M^{3}$. Let $p=0$ or a prime. If $M^{3}$ is nonorientable assume $p=2$. Then $(1) b(X)$ is definable by free 3-manifolds if and only if $\pi_{1}(X)$ is free, and $(2) h(X)$ is definable by homotopy handlebodies if and only if $\pi_{1}(X)$ is free and $X$ bas property uv $\left(2,0 ; Z_{p}\right)$.

Proof, Let $N^{3}$ be a compact, polyhedral 3-manifold in Int $M^{3}$ such that $h(X) \subset$ Int $N^{3}$ and $N^{3}$ retracts onto $h(X)$. Note the inclusion $b(X) \subset N^{3}$ induces monomorphisms on homology and homotopy groups. We will prove (1). The proof of $(2)$ is similar. If $h(X)$ is definable by free 3 -manifolds then $\pi_{1}(X)$ is free. By Lemma 18 and the Addendum, $X$ has properties uv $\left(1, m ; Z_{p}\right)$ and uv $\left(2, l ; Z_{p}\right)$ for $m=\rho H_{1}\left(X ; Z_{p}\right)$ and $l=\rho H_{2}\left(X ; Z_{p}\right)$. Again by Lemma 18 we see $X$ has property UVF. By Theorem 15 we are done. 
Corollary 20. Suppose $X$ is a continuum and $h_{1}, h_{2}$ are topological embeddings of $X$ into the interiors of nonclosed, piecewise-linear 3-manifolds $M_{1}^{3}, M_{2}^{3}$, respectively. Let $p=0$ or a prime. If either $M_{1}^{3}$ or $M_{2}^{3}$ is nonorientable, assume $p=2$. Then (1) If $X$ is locally 1 -connected and $H_{1}\left(X ; Z_{p}\right)$ and $H_{2}\left(X ; Z_{p}\right)$ are finitely generated, then $b_{1}(X)$ is definable by free 3-manifolds if and only if $b_{2}(X)$ is definable by free 3-manifolds, and (2) If $X$ is locally 0-connected and $H_{1}\left(X ; Z_{p}\right)$ is finitely generated, then $h_{1}(X)$ is definable by homotopy handlebodies if and only if $h_{2}(X)$ is definable by homotopy handlebodies.

Proof. Considering (1) we see that by the addendum to Lemma $18, X$ has properties uv $\left(1, m ; Z_{p}\right)$ and uv $\left(2, l ; Z_{p}\right)$ for $m=\rho H_{1}\left(X ; Z_{p}\right)$ and $l=\rho H_{2}\left(X ; Z_{p}\right)$. If either $h_{1}(X)$ or $h_{2}(X)$ is definable by free 3 -manifolds, then $X$ has property UVF. By Theorem 15, (1) holds.

For (2) note that $X$ has property uv $\left(1, m ; Z_{p}\right)$ where $m=\rho H_{1}\left(X ; Z_{p}\right)$. If either $h_{1}(X)$ or $h_{2}(X)$ is definable by homotopy handlebodies then $X$ has properties UVF and uv $\left(2,0 ; Z_{p}\right)$. Hence by Corollary 16, (2) holds.

\section{REFERENCES}

1. Steve Armentrout, Decompositions of $E^{3}$ with a compact 0 -dimensional set of nondegenerate elements, Trans. Amer. Math. Soc. 123 (1966), 165-177. MR 33 \#3279.

2. R. H. Bing, Tame Cantor sets in $E^{3}$, Pacific J. Math. 11 (1961), 435-446. MR 24 \#A539.

3. K. Borsuk, The ory of shape, Lecture Note Series, no. 28, Matematisk Institut, Aarhus Universitet, Aarhus, 1971, 145 pp. MR 45 \#2679.

4. H. G. Bothe, Ein eindimensionales Kompactum in $E^{3}$, das sich nicht lagetreu in die Mengersche Universalkurve eingebetten lässt, Fund. Math. 54 (1964), 251-258. MR 29 \#6470.

5. - A tangled 1-dimensional continuum in $E^{3}$ which has the cube-withhandles property, Bull. Acad. Polon. Sci. Sér. Sci. Math. Astronom. Phys. 20 (1972), 481-486.

6. R. Craggs, Cannonical neighborhoods for topologically embedded polyhedra, Trans. Amer. Math. Soc. (to appear).

7. W. Haken, Some results on surfaces in 3-manifolds, Studies in Modern Topology, Math. Assoc. Amer., (distributed by Prentice-Hall, Englewood Cliffs, N. J.), 1968, pp. 39-98. MR $36 \# 7118$. notes).

8. —_ Erratum for "Some results on surfaces in 3-manifolds" (mimeographed

9. Sze-Tsen Hu, Theory of retracts, Wayne State Univ. Press, Detroit, Mich., 1965. MR $31 \# 6202$.

10. W. Hurewicz and H. Wallman, Dimension theory, Princeton Math. Series, vol. 4, Princeton Univ. Press, Princeton, N. J., 1941. MR 3, 312.

11. S. Lefschetz, On compact spaces, Ann. of Math. 32 (1931), 521-538.

12. W. Magnus, A. Karrass and D. Solitar, Combinatorial group theory: Presentations of groups in terms of generators and relations, Pure and Appl. Math., vol. 13, Interscience, New York, 1966. MR 34 \#7617. 
13. S. Mardešić and J. Segal, Equivalence of the Borsuk and ANR-system approach to shapes, Fund. Math. 72 (1971), no. 1, 61-68. MR 46 \#850.

14. - Shapes of compacta and ANR-systems, Fund. Math. 72 (1971), no. 1, 4159. MR $45 \# 7686$.

15. W. S. Massey, Algebraic topology: An introduction, Harcourt, Brace and World, New York, 1967. MR 35 \#2271.

16. D. R. McMillan, Jr., A criterion for cellularity in a manifold. II, Trans. Amer. Math. Soc. 126 (1967), 217-224. MR 34 \#8392.

17. - Neighborhoods of surfaces in 3-manifolds, Michigan Math. J. 14 (1967), 161-170. MR $35 \# 3643$.

18. - Compact, acyclic subsets of three manifolds, Michigan Math. J. 16 (1969), 129-136. MR 39 \#4822.

19. —-, Acyclicity in three-manifolds, Bull. Amer. Math. Soc. 76 (1970), 942964. MR $42 \# 5269$.

20. - Boundary-preserving mappings of 3-manifolds, Topology of Manifolds (Proc. Inst., Univ. of Georgia, Athens, Ga., 1969), Markham, Chicago, Ill., 1970, pp. 161175. MR $43 \# 2723$.

21. - UV properties and related topics, Lecture notes compiled by Brian J. Smith, Florida State University, Tallahassee, Florida.

22. - Non-planar embeddings of planar sets in $E^{3}$ (preprint).

23. D. R. McMillan, Jr. and Harry Row, Tangled embeddings of one-dimensional continua, Proc. Amer. Math. Soc. 22 (1969), 378-385. MR 39 \#7571.

24. J. Milnor, $A$ unique decomposition theorem for 3-manifolds, Amer. J. Math. 84 (1962), 1-7. MR $25 \# 5518$.

25. E. H. Spanier, Algebraic topology, McGraw-Hill, New York, 1966. MR 35 \#1007.

26. J. R. Stallings, On the loop theorem, Ann. of Math. (2) 72 (1960), 12-19. MR $22 \# 12526$.

27. - On fibering certain 3-manifolds, Topology of 3-Manifolds and Related Topics (Proc. Univ. of Georgia Inst., 1961), Prentice-Hall, Englewood Cliffs, N. J., 1962, pp. 95-100. MR $28 \# 1600$.

28. E. C. Zeeman, Seminar on combinatorial topology, Inst. Hautes Études Sci. Paris (1963) (mimeograph notes).

DEPARTMENT OF MATHEMATICS, UNIVERSITY OF TENNESSEE, KNOXVILLE, TENNESSEE 37916 